SNUTP 97-047

\title{
In-Medium Effective Axial-Vector Coupling Constant
}

\author{
Tae-Sun Park \\ Department of Physics, Hanyang University, Seoul 133-791, Korea \\ Hong Jung \\ Department of Physics, Sookmyung Women's University, Seoul 140-742, Korea \\ Dong-Pil Min \\ Department of Physics and Center for Theoretical Physics, \\ Seoul National University, Seoul 151-742, Korea
}

\begin{abstract}
The axial-vector coupling constant in nuclear medium is systematically studied using chiral perturbation theory. For normal nuclear matter, the first non-trivial corrections are estimated based on the independent particle approximation taking into account nuclear correlations in nuclear medium. The one-pion-exchange (OPE) two-body interactions turn out to enhance the coupling constant by $\delta g_{A} / g_{A} \simeq(0.09 \sim 0.15)$. The role of the four-nucleon contact interaction, whose low-energy constants are yet to be determined, is analyzed in connection with the empirical quenching factor of $\delta g_{A} / g_{A} \simeq-0.2$ in the presence of the nuclear short-range correlation. Conventional approaches are discussed in the light of our result.
\end{abstract}


The nucleon's axial-vector coupling constant in nuclear medium $g_{A}^{*}$ is empirically known to be quenched by the amount of $\frac{\delta g_{A}}{g_{A}} \equiv \frac{g_{A}^{*}-g_{A}}{g_{A}} \simeq-0.2$ [1]. Since the in-medium quenching is very important for various places in nuclear physics, there have been many efforts for its explanation[2]. One well-known example is the question whether or not the pion condensation is practically possible at a low nuclear density [3]. Indeed, wide discussions on the change of matter properties at the extreme condition take place in conjunction with CERN-CERES, RHIC and CEBAF experiments.

In most conventional approaches, a major contribution to the quenching of the axialvector coupling in nuclear medium is from the renormalization due to the $\Delta$-hole screening induced by the short-range interaction in $N N \leftrightarrow N \Delta$ (Hartree) channel with the net contribution from other mechanisms presumed to be negligible due to the mutual cancellation and/or due to the famous nuclear correlation [4]. Also, some fascinating efforts have been given to figure out the medium effects on physical quantities by taking the tree order only with some scaled parameters of chiral Lagrangian, which is known as "Brown-Rho scaling" [5]. We wish to understand the conventional approaches based on a well-defined systematic method.

In this letter, we aim to study the nucleon's axial-vector coupling in nuclear medium in a systematic way using chiral perturbation theory generalized to many nucleon systems 6, 6]. Chiral perturbation theory has been successful in explaining the nuclear force[8] and the so-called chiral filter phenomena [7, 9, 10]. In order to extract the axial-vector coupling constant, the relevant quantity is the transition amplitude of the Gamow-Teller operator, that is, the space part of the axial-vector current in the limit $k^{\mu} \rightarrow 0$ where $k^{\mu}$ is the momentum carried by the external field. For a single nucleon in free space, the GamowTeller operator is given as

$$
g_{A} \tau^{ \pm} \vec{\sigma}
$$

In nuclear medium, the axial-vector current is modified by the presence of other nucleons, and how the transition amplitude of the Gamow-Teller operator changes is what we are interested in.

The chiral power counting rule for $A$-nucleon processes [6] is that for a Feynman graph with $V_{i}$ vertices of type $i, L$ loops, and $C$ separately connected pieces, the number of powers of $Q$ which is the typical low momentum scale is given as

$$
\nu=4-A-2 C+2 L+\sum_{i} V_{i} \Delta_{i} \text { with } \Delta_{i}=d_{i}+\frac{n_{i}}{2}-2
$$

where $n_{i}$ is the number of nucleon lines and $d_{i}$ is the number of derivatives or powers of $m_{\pi}$ at the $i$-type vertex. Chiral symmetry guarantees $\Delta_{i} \geq 0$ [6], so the effective Lagrangian can be ordered according to $\Delta_{i}$ :

$$
\mathcal{L}_{\text {eff }}=\mathcal{L}_{0}+\mathcal{L}_{1}+\mathcal{L}_{2}+\cdots
$$

where $\mathcal{L}_{n}$ has vertices with $\Delta_{i}=n$. However, in the presence of an external field, the lower bound on $\Delta_{i}$ is modified to $\Delta_{i} \geq-1$ because of the gauge invariant derivative coupling 11 . 
In this work, we take the nucleon and the pion as pertinent degrees of freedom. The contribution of $\Delta$ will be properly encoded in some of the low-energy constants. Then, the leading order Lagrangian is

$$
\begin{aligned}
\mathcal{L}_{0}= & \bar{B}\left[i v \cdot D+2 i g_{A} S \cdot \Delta\right] B-\frac{1}{2} \sum_{A} C_{A}\left(\bar{B} \Gamma_{A} B\right)^{2} \\
& +f_{\pi}^{2} \operatorname{Tr}\left(i \Delta^{\mu} i \Delta_{\mu}\right)+\frac{f_{\pi}^{2}}{4} \operatorname{Tr}\left(\chi_{+}\right)
\end{aligned}
$$

with

$$
\begin{aligned}
D_{\mu} N & =\left(\partial_{\mu}+\Gamma_{\mu}\right) N \\
\Gamma_{\mu} & =\frac{1}{2}\left[\xi^{\dagger}, \partial_{\mu} \xi\right]-\frac{i}{2} \xi^{\dagger} \mathcal{R}_{\mu} \xi-\frac{i}{2} \xi \mathcal{L}_{\mu} \xi^{\dagger}, \\
\Delta_{\mu} & =\frac{1}{2}\left[\xi^{\dagger}, \partial_{\mu} \xi\right]+\frac{i}{2} \xi^{\dagger} \mathcal{R}_{\mu} \xi-\frac{i}{2} \xi \mathcal{L}_{\mu} \xi^{\dagger}, \\
\chi_{+} & =\xi^{\dagger} \chi \xi^{\dagger}+\xi \chi^{\dagger} \xi
\end{aligned}
$$

where $\mathcal{R}_{\mu}=\frac{\tau^{a}}{2}\left(\mathcal{V}_{\mu}^{a}+\mathcal{A}_{\nu}^{a}\right)$ and $\mathcal{L}_{\mu}=\frac{\tau^{a}}{2}\left(\mathcal{V}_{\mu}^{a}-\mathcal{A}_{\nu}^{a}\right)$ denote the external gauge fields, $\chi$ is proportional to the quark mass matrix and it becomes, if we neglect the small isospinsymmetry breaking, $\chi=m_{\pi}^{2}$ in the absence of the external scalar and pseudo-scalar gauge field, and

$$
\xi=\sqrt{\Sigma}=\exp \left(i \frac{\vec{\tau} \cdot \vec{\pi}}{2 f_{\pi}}\right)
$$

For convenience, we will work in a reference frame in which the four velocity $v^{\mu}$ and the spin operator $S^{\mu}$ are

$$
v^{\mu}=(1, \overrightarrow{0}) \quad \text { and } \quad S^{\mu}=\left(0, \frac{\vec{\sigma}}{2}\right)
$$

From [12] and [13] terms can be written as

$$
\begin{aligned}
\mathcal{L}_{1}= & \bar{B}\left(\frac{v^{\mu} v^{\nu}-g^{\mu \nu}}{2 m_{N}} D_{\mu} D_{\nu}+4 c_{3} i \Delta \cdot i \Delta+\left(2 c_{4}+\frac{1}{2 m_{N}}\right)\left[S^{\mu}, S^{\nu}\right]\left[i \Delta_{\nu}, i \Delta_{\nu}\right]\right) B \\
& -4 i d_{1} \bar{B} S \cdot \Delta B \bar{B} B+2 i d_{2} \epsilon^{a b c} \epsilon_{\mu \nu \lambda \delta} v^{\mu} \Delta^{\nu, a} \bar{B} S^{\lambda} \tau^{b} B \bar{B} S^{\delta} \tau^{c} B+\cdots,
\end{aligned}
$$

where $\epsilon_{0123}=1, \Delta_{\mu}=\frac{\tau^{a}}{2} \Delta_{\mu}^{a}$, and only those terms relevant for our study are shown explicitly.

The leading contribution to the transition amplitude of the Gamow-Teller operator is given by the one-body currents $(L=0, C=A)$ with one $\Delta_{i}=-1$ interaction $(\nu=3-3 A)$. The one-body currents, including corrections to all order, are entirely determined by the experiment, $g_{A} \simeq 1.26$.

The first corrections due to the nuclear medium are given by tree graphs of two-body currents $(L=0, C=A-1)$ with one $\Delta_{i}=-1$ interaction and interactions with $\Delta_{i}=0$

* Our definition of the pion field is different from that used in Ref. [13]: We should attach a minus sign for the pion field of Ref. 13] and then make the interaction form of Ref. 13] manifestly Lorentz-invariant and chiral-invariant. 


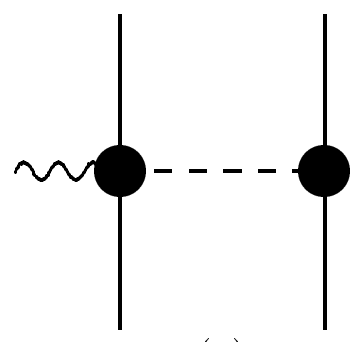

(a)

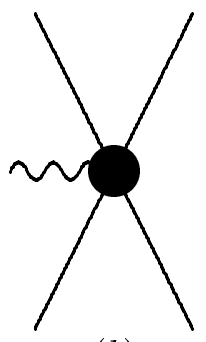

(b)

Figure 1: Generic graphs contributing to the exchange current: the graph $(a)$ is the OPE graph and the graph $(b)$ is the four-fermion contact graph. The large filled circles denote the one-nucleon and one-pion irreducible graphs and the solid (dashed) lines the renormalized nucleon (pion) propagators. The renormalization has been taken up to $1 / m_{N}$ correction.

$(\nu=5-3 A)$. Since there are no $\Delta_{i}=-1$ four-nucleon contact interactions, at order $\nu=$ $5-3 A$ we have only one-pion-exchange graphs, but they do not contribute to the GamowTeller operator because the currents from one-pion-exchange graphs are proportional to $v^{\mu}=(1, \overrightarrow{0})$. Thus, the leading order medium corrections to the space part of the axial-vector currents are kinematically suppressed. Therefore the higher order contributions including the vertex corrections as well as the short-range contributions become important 9]. This situation is contrary to the case of the axial-charge in which the one-body currents are suppressed while the leading two-body currents are not [0, 10].

The first non-trivial medium corrections come from tree-graphs of two-body currents with $\Delta_{i}=0$ interactions $(\nu=6-3 A)$. More specifically, we can have one-pion-exchange (OPE) graphs (Fig. 1(a)) and four-nucleon contact graphs (Fig. 1(b)). In the limit when momentum carried by external field goes to zero, the space part of the axial-vector currents reads

$$
\begin{aligned}
\vec{J}_{5}^{ \pm}(\vec{q})=- & \frac{g_{A}}{m_{N} f_{\pi}^{2}} \frac{1}{m_{\pi}^{2}+\vec{q}^{2}}\left\{\frac{i}{2}\left(\tau_{1} \times \tau_{2}\right)^{ \pm}\left(\vec{p}_{1} \vec{\sigma}_{2} \cdot \vec{q}+\vec{p}_{2} \vec{\sigma}_{1} \cdot \vec{q}\right)\right. \\
& +2 \hat{c}_{3} \vec{q}\left(\tau_{1}^{ \pm} \vec{\sigma}_{1} \cdot \vec{q}+\tau_{2}^{ \pm} \vec{\sigma}_{2} \cdot \vec{q}\right) \\
& \left.\quad+\left(\hat{c}_{4}+\frac{1}{4}\right)\left(\tau_{1} \times \tau_{2}\right)^{ \pm}\left(\vec{\sigma}_{1} \times \vec{q} \vec{\sigma}_{2} \cdot \vec{q}-\vec{\sigma}_{2} \times \vec{q} \vec{\sigma}_{1} \cdot \vec{q}\right)\right\} \\
- & \frac{2 g_{A}}{m_{N} f_{\pi}^{2}}\left[\hat{d}_{1}\left(\tau_{1}^{ \pm} \vec{\sigma}_{1}+\tau_{2}^{ \pm} \vec{\sigma}_{2}\right)-\hat{d}_{2}\left(\tau_{1} \times \tau_{2}\right)^{ \pm}\left(\vec{\sigma}_{1} \times \vec{\sigma}_{2}\right)\right]
\end{aligned}
$$

where $\vec{q} \equiv \vec{p}_{2}^{\prime}-\vec{p}_{2}$, the subscript $l$ of $\tau_{l}^{ \pm}=\frac{1}{2}\left(\tau_{l}^{1} \pm i \tau_{l}^{2}\right), \vec{\sigma}_{l}$, and $\vec{p}_{l} \equiv \frac{1}{2}\left(\vec{p}_{l}+\vec{p}_{l}^{\prime}\right)$ is the particle index, $l=1$ for the first nucleon and $l=2$ for the second nucleon, and we have defined dimensionless parameters $\hat{c}_{i}$ 's and $\hat{d}_{i}$ 's by

$$
c_{i} \equiv \frac{1}{m_{N}} \hat{c}_{i} \quad \text { and } \quad d_{i} \equiv \frac{g_{A}}{m_{N} f_{\pi}^{2}} \hat{d}_{i} .
$$

The first term in the curly bracket comes from $\frac{1}{2 m_{N}} \bar{B} \vec{D}^{2} B$ of eq. (8) and will be referred to as the kinetic term. The low-energy constants $c_{3}$ and $c_{4}$ have been determined from the 
experiment by Bernard et. al. [12]:

$$
\begin{aligned}
& c_{3}=-5.29 \pm 0.25 \mathrm{GeV}^{-1}, \\
& c_{4}=3.63 \pm 0.10 \mathrm{GeV}^{-1} .
\end{aligned}
$$

These values of $c_{3}$ and $c_{4}$ are almost saturated by the resonance-exchange contributions:

$$
\begin{aligned}
& c_{3}^{\text {Res }}=c_{3}^{\Delta}+c_{3}^{S}+c_{3}^{R}=(-3.83-1.40-0.06) \mathrm{GeV}^{-1}=-5.29 \mathrm{GeV}^{-1}, \\
& c_{4}^{\text {Res }}=c_{4}^{\Delta}+c_{4}^{\rho}+c_{4}^{R}=(1.92+1.63+0.12) \mathrm{GeV}^{-1}=3.67 \mathrm{GeV}^{-1}
\end{aligned}
$$

where the superscripts $\Delta, \rho, S$ and $R$ denote the contributions from the exchange of $\Delta(1232)$, $\rho$-mesons, scalar-mesons and the Roper resonance, respectively. In terms of the dimensionless constants $\hat{c}_{3}$ and $\hat{c}_{4}$, the above eq.(11) gives us

$$
\begin{aligned}
& \hat{c}_{3} \simeq-4.97 \pm 0.23, \\
& \hat{c}_{4} \simeq 3.41 \pm 0.09 .
\end{aligned}
$$

For the low-energy constants $\hat{d}_{1}$ and $\hat{d}_{2}$, however, we do not have any empirical information yet. So we simply take these constants as free parameters in this work.

Now we are in the position to calculate the matrix elements of the space part of the two-body axial-vector currents. First we estimate the matrix elements using the independent particle approximation, i.e., we take the initial (final) wavefunction as the simple product of one-nucleon state $\alpha(\beta)$ and the Fock state $|F\rangle$ which describes the Fermi sea,

$$
|F\rangle=\prod_{h \in F} a_{h}^{\dagger}|v a c\rangle,
$$

where $h$ denotes a state in the Fermi sea. Since the momentum carried by external field is zero, only those states on the Fermi surface can contribute to the matrix elements and we have

$$
\vec{p}_{\beta}=\vec{p}_{\alpha} \equiv \vec{p} \text { with } \quad|\vec{p}|=k_{F} .
$$

Then the two-body matrix elements relevant for the axial-vector coupling constant are

$$
\left\langle\beta ; F\left|J_{5}^{i, \pm}(\vec{q})\right| \alpha ; F\right\rangle=\sum_{h \in F}\left[\left\langle\beta, h\left|J_{5}^{i, \pm}(\vec{q})\right| \alpha, h\right\rangle-\left\langle\beta, h\left|J_{5}^{i, \pm}(\vec{q})\right| h, \alpha\right\rangle\right]
$$

where the first term is the direct (or Hartree) term and the second is the exchange (or Fock) term. We average over the the direction of the probed nucleons in order to obtain the in-medium axial-vector coupling constant,

$$
\int \frac{d \Omega_{\hat{p}}}{4 \pi}\left\langle\beta ; F\left|J_{5}^{i, \pm}(\vec{q})\right| \alpha ; F\right\rangle \equiv \delta g_{A} \tau_{\beta \alpha}^{ \pm} \sigma_{\beta \alpha}^{i}, \quad \delta g_{A}=\delta^{H} g_{A}+\delta^{F} g_{A}
$$

where $\tau_{\beta \alpha}^{ \pm} \equiv\left\langle t_{\beta}\left|\tau^{ \pm}\right| t_{\alpha}\right\rangle, \sigma_{\beta \alpha}^{j} \equiv\left\langle s_{\beta}\left|\sigma^{j}\right| s_{\alpha}\right\rangle$ with $t_{\alpha}\left(t_{\beta}\right)$ and $s_{\alpha}\left(s_{\beta}\right)$ being the quantum numbers for the third components of the isospin and the spin of a state $\alpha(\beta)$ respectively, and we 


\begin{tabular}{|c||rrrrr|r|}
\hline & $\Delta$ & $\rho$ & $S$ & $N^{*}$ & kin. & Experiment \\
\hline Zero-range & -0.386 & -0.164 & -0.070 & -0.015 & -0.013 & -0.672 \\
Finite-range & 0.078 & 0.033 & 0.014 & 0.003 & 0.018 & 0.151 \\
\hline Sum & -0.308 & -0.131 & -0.056 & -0.012 & 0.004 & -0.521 \\
\hline
\end{tabular}

Table 1:

One-pion-exchange (Fig. 1(a)) contributions to the $\delta g_{A} / g_{A}$. The contributions from the resonance-exchanges through the $\pi \mathcal{A} N N$ vertex has been listed in column $2 \sim 5$. The last column represents all the contributions with $\hat{c}$ 's fixed from the $\pi N$ scattering data 12 and the kinetic term. The second row shows zero-ranged (in position space) contributions, that is, those which are constant in momentum space, the third row does finite-ranged contributions and the last row does the sum of the two.

have separated the Hartree contribution $\left(\delta^{H} g_{A}\right)$ from the Fock contribution $\left(\delta^{F} g_{A}\right)$. Note that the Hartree term for the OPE contribution is identically zero because it involves only $\vec{q}=\overrightarrow{0}$ and the corresponding axial-vector current vanishes, thus we have

$$
\frac{\delta^{H} g_{A}}{g_{A}}=-2 \hat{d}_{1} \frac{\rho}{m_{N} f_{\pi}^{2}}
$$

with $\rho=\frac{2 k_{F}^{3}}{3 \pi^{2}}$ being the density of symmetric nuclear matter. It is also quite straightforward to evaluate the Fock contribution,

$$
\frac{\delta^{F} g_{A}}{g_{A}}=\frac{4}{m_{N} f_{\pi}^{2}} \int^{k_{F}} \frac{d^{3} \vec{k}}{(2 \pi)^{3}}\left[\frac{\frac{1}{24}\left(\vec{k}^{2}-\vec{p}^{2}\right)+\frac{1}{3}\left(\hat{c}_{3}-2 \hat{c}_{4}-\frac{1}{2}\right)(\vec{k}-\vec{p})^{2}}{m_{\pi}^{2}+(\vec{k}-\vec{p})^{2}}+\hat{d}_{1}+2 \hat{d}_{2}\right] .
$$

Performing the momentum integral over the Fermi sea, we have

$$
\frac{\delta^{F} g_{A}}{g_{A}}=\frac{\rho}{m_{N} f_{\pi}^{2}}\left\{\frac{k_{F}^{2}}{6 m_{\pi}^{2}} H_{1}\left(\frac{k_{F}}{m_{\pi}}\right)+\frac{1}{3}\left(\hat{c}_{3}-2 \hat{c}_{4}+\frac{1}{4}\right)\left[1-H_{0}\left(\frac{k_{F}}{m_{\pi}}\right)\right]+\hat{d}_{1}+2 \hat{d}_{2}\right\}
$$

with

$$
\begin{aligned}
& H_{0}(x)=\frac{3}{2 x^{2}}\left[1-\frac{1}{x} \tan ^{-1}(2 x)+\frac{1}{4 x^{2}} \log \left(1+4 x^{2}\right)\right], \\
& H_{1}(x)=\frac{3}{8 x^{4}}\left[1+2 x^{2}+\left(1+\frac{1}{4 x^{2}}\right) \log \left(1+4 x^{2}\right)\right] .
\end{aligned}
$$

For normal nuclear matter, $k_{F} \simeq 1.36 \mathrm{fm}^{-1}, \rho \simeq 0.17 \mathrm{fm}^{-3}$, thus leading to $H_{0}\left(\frac{k_{F}}{m_{\pi}}\right) \simeq 0.20$ and $\frac{k_{F}^{2}}{m_{\pi}^{2}} H_{1}\left(\frac{k_{F}}{m_{\pi}}\right) \simeq 0.55$.

We now discuss the numerical outcome on the quenching factor $\delta g_{A} / g_{A}$, though naive, firstly without taking the correlation into account. In Table 1, we have listed the contributions from resonance-exchanges through the $\pi \mathcal{A} N N$ vertex using the estimates of Ref.[12], of which relevant diagrams are illustrated in Fig. 2. We have separated the zerorange part of the OPE contribution from the finite-range part. The former is subject to the 


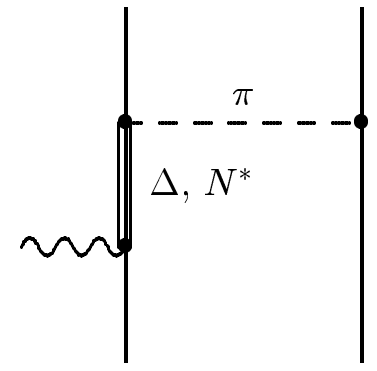

$(a)$

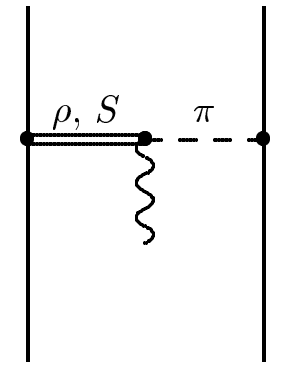

$(b)$

Figure 2: The resonance-exchange graphs which contribute to Fig. 1(a).

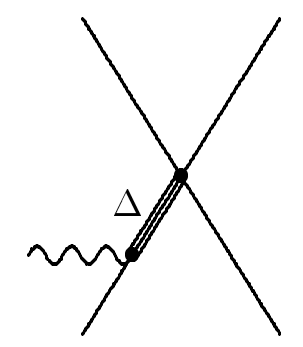

Figure 3: A graph contributing to Fig. 1(b).

short-range correlation (SRC) between nucleons which we will discuss below. Note that the last column is obtained taking the values of $c_{3}$ and $c_{4}$ as determined from the experiment in Ref.[12]; it is not just the sum of the contributions from the resonance-exchanges and the kinetic term. The naive quenching of the axial-vector coupling due to the OPE graph (Fig. $1(a)$ ) is by the amount of $\delta g_{A} / g_{A} \simeq-0.52$. In order to reproduce the experimental coupling constant $g_{A}^{*} \simeq 1.0$, the four-fermion contact graph (Fig. 1(b)) needs to enhance the coupling by about 0.32 . Meanwhile, the four-nucleon contact interaction contains implicitly the contribution of Fig. 3, which is the main part of the conventional mechanism of the quenching. This contradictory behavior, however, disappears when we incorporate the SRC.

For the purpose of including the SRC, it is much more convenient to work in the position space. In regard to the SRC, it is worth paying attention to the conventional wisdom of applying the nuclear correlation only to the exchange (Fock) channel. The direct (Hartree) contribution is effectively from the expectation value of one-body operators. Therefore the Hartree contribution is the same as eq.(17). Incorporating the SRC for the Fock term by cutting-off the contribution from $r<r_{c}$, we have

$$
\frac{\delta^{F} g_{A}}{g_{A}}=\frac{m_{\pi}^{3} \rho}{m_{N} f_{\pi}^{2}} \int_{r_{c}}^{\infty} d r \frac{r j_{1}\left(k_{F} r\right)}{k_{F}}\left[\frac{k_{F}}{2 m_{\pi}} j_{1}\left(k_{F} r\right) Y_{1}\left(m_{\pi} r\right)-\left(\hat{c}_{3}-2 \hat{c}_{4}+\frac{1}{4}\right) j_{0}\left(k_{F} r\right) Y_{0}\left(m_{\pi} r\right)\right]
$$

where $j_{0}(x)=\frac{\sin (x)}{x}, j_{1}(x)=\frac{\sin (x)}{x^{2}}-\frac{\cos (x)}{x}, Y_{0}(x)=\frac{\mathrm{e}^{-x}}{x}$ and $Y_{1}(x)=\left(1+\frac{1}{x}\right) Y_{0}(x)$. In Table 2 , the Fock contribution is calculated for several values of $r_{c}$ and the Hartree contribution is adjusted to give the empirical quenching of $\delta g_{A} / g_{A} \simeq-0.2$. We find that the OPE 


\begin{tabular}{|l|c|c||c|c|}
\hline & Fock (OPE) & Hartree (Contact) & $\hat{d}_{1}$ & $g_{N \Delta}^{\prime}$ \\
\hline Without OPE & 0 & -0.21 & 0.64 & 0.25 \\
\hline$\left(r_{c}=0^{+}\right)$ & 0.15 & -0.36 & 1.11 & 0.43 \\
$\left(r_{c}=0.4 \mathrm{fm}\right)$ & 0.13 & -0.34 & 1.04 & 0.40 \\
$\left(r_{c}=0.7 \mathrm{fm}\right)$ & 0.09 & -0.30 & 0.94 & 0.37 \\
\hline
\end{tabular}

Table 2:

Fock (2nd column) and Hartree (3rd column) contributions to $\delta g_{A} / g_{A}$ at normal nuclear density with various cut-offs. The $\hat{d}_{1}$ is fitted to give $\delta g_{A} \simeq-0.26$. In the second row, we have also listed the result without OPE.

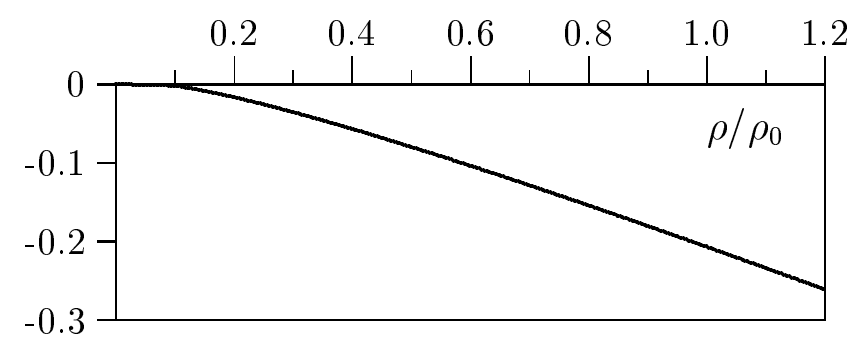

Figure 4: $\delta g_{A} / g_{A}$ with respect to the density. The cut-off is taken as $r_{c}=0^{+}$.

contribution is of sizable importance, i.e., $\frac{\delta g_{A}}{g_{A}} \simeq(0.09 \sim 0.15)$. Nevertheless, the quenching comes mainly from the four-nucleon contact interaction. The importance of the short-range interaction in the quenching of the axial-vector coupling is a well-known observation in the conventional approaches. In this work, such an observation is naturally understood based on chiral perturbation theory.

The density dependence of $\delta g_{A} / g_{A}$ with $r_{c}=0^{+}$has been plotted in Fig. 4 . The leading non-trivial medium correction is roughly linear in the density and this aspect is mainly due to the fact that the zero-range interaction accounts for the quenching for the most part.

For comparison with the conventional approach, we estimate the corresponding LandauMigdal parameter $g_{N \Delta}^{\prime}$ in the effective $N N \leftrightarrow N \Delta$ potential by identifying the potential from the four-nucleon contact interaction with the phenomenological one as in Ref. 4 :

$$
\hat{d}_{1}=\frac{4 m_{N} f_{\pi}^{2} f_{\Delta}^{2}}{9\left(m_{\Delta}-m_{N}\right) m_{\pi}^{2}} g_{N \Delta}^{\prime} .
$$

Our result shown in Table $2, g_{N \Delta}^{\prime}=(0.37 \sim 0.43)$, lies well within the range of the empirical value with $\frac{f_{\Delta}^{2}}{4 \pi} \simeq 0.32[2]$.

In more realistic calculations, surface effects as well as short-range correlations between nucleons will be incorporated in the wavefunction. At present, such a wavefunction for nuclear matter or finite nuclei has not been obtained using chiral perturbation theory. 
Thus, it would be interesting to study how the above features in the independent particle approximation change in more realistic approximations. Furthermore we believe it worth investigating the nuclear medium modification of the physical quantities such as the nucleon mass and the pion decay constant in relation with the "Brown-Rho scaling" [5] in the light of present work.

\section{ACKNOWLEDGMENTS}

We are very grateful to Mannque Rho for valuable comments. This work was supported in part by Korea Ministry of Education, Korea (BSRI-96-2418, BSRI-96-2441) and in part by Korea Science and Engineering Foundation through Center for Theoretical Physics, SNU.

\section{References}

[1] B. H. Wildenthal, M. S. Curtin and B. A. Brown, Phys. Rev. C28 (1983) 1343; D. H. Wilkinson in "Physics with Heavy Ions and Mesons", ed. R. Balian, M. Rho and G. Ripka (North Holland, Amsterdam, 1978); B. Buck and S. M. Perez, Phys. Rev. Lett. 50 (1983) 1975; K. Langanke, D. J. Dean, P. B. Radha, Y. Alhassid and S. E. Koonin, "Shell-model Monte Carlo studies of fp-shell nuclei", nucl-th/9504019.

[2] See Pions and Nuclei by T. Ericson and W. Weise (Clarendon, Oxford, 1988) and references therein.

[3] Mesons in Nuclei, ed. M. Rho and D. Wilkinson (North Holland, Amsterdam-New York-Oxford, 1979).

[4] K. Ohta and M. Wakamatsu, Nucl. Phys. A234 (1974) 445; M. Rho, Nucl. Phys. A231 (1974) 493.

[5] G.E. Brown and M. Rho, Phys. Rev. Lett. 66 (1991) 2720; M. Rho, J. Korean Phys. Soc. 29 (1996) S337; B. Friman and M. Rho, Nucl. Phys. A606 (1996) 303.

[6] S. Weinberg, Phys. Lett. B251 (1990) 288; Nucl. Phys. B363 (1991) 3; Phys. Lett. B295 (1992) 114.

[7] T-S. Park, D.-P. Min and M. Rho, Phys. Rep. 233 (1993) 341.

[8] C. Ordonez, L. Ray, and U. van Kolck, Phys. Rev. Lett. 72 (1994) 1982; Phys. Rev. C53 (1996) 2086; U. van Kolck, Phys. Rev. C49 (1994) 2932. 
[9] K. Kubodera, J. Delorme and M. Rho, Phys. Rev. Lett. 40 (1978) 755.

[10] T.-S. Park, D.-P. Min and M. Rho, Phys. Rev. Lett. 74 (1995) 4153; T.-S. Park, D.-P. Min and M. Rho, Nucl. Phys. A 596 (1996) 515; T.-S. Park, I. S. Towner and K. Kubodera, Nucl. Phys. A579 (1994) 381.

[11] M. Rho, Phys. Rev. Lett. 66 (1991) 1275

[12] V. Bernard, N. Kaiser and Ulf-G. Meissner, Nucl. Phys. A615 (1997) 483.

[13] T. D. Cohen, J. L. Friar, G. A. Miller and U. van Kolck, Phys. Rev. C53 (1996) 2661. 